\title{
Uso de cenário com pessoas com deficiência e seus familiares no ensino odontológico
}

\author{
Andréa Paula Fregoneze*; João Armando Brancher*; Dilmeire Sant'Anna Ramos Vosgerau**; Aline \\ Cadena Von Bahten***
}

* Professor, Departamento de Odontologia, Pontifícia Universidade Católica do Paraná

** Professor, Programa de Pós-Graduação em Educação, Pontifícia Universidade Católica do Paraná

*** Professor, Escola de Medicina, Pontifícia Universidade Católica do Paraná

Recebido em 30/07/2019. Aprovado em 20/06/2020.

\begin{abstract}
RESUMO
O objetivo deste estudo foi usar um cenário envolvendo pessoas com deficiência, seus familiares e estudantes de Odontologia para avaliar a percepção dos estudantes a respeito das necessidades destas pessoas. Trata-se de um estudo qualitativo transversal no qual participaram 60 estudantes de Odontologia matriculados na disciplina de Clínica Integrada Para Pacientes com Necessidades Especiais. Formaramse grupos focais que receberam um questionário semiestruturado com questões que exploraram aspectos sobre o atendimento de pessoas com deficiência e da rotina destas pessoas. Na sequência utilizou-se um cenário no qual estudantes interagiram com pessoas com deficiência e seus familiares. Na última etapa os grupos formularam hipóteses a respeito do atendimento destes pacientes. Os dados coletados nos três momentos foram agrupados em categorias e temas e analisados no software Atlas Ti. Os principais resultados obtidos foram agrupados em categorias de acordo com a percepção dos estudantes antes e depois da criação do cenário. Os dados obtidos após a análise qualitativa revelam que antes da criação do cenário os estudantes se colocaram no centro da discussão argumentando que atender pessoas com deficiência seria uma oportunidade para desenvolver suas habilidades, sem se preocupar com a pessoa com deficiência em si. Após a criação do cenário parece ter havido uma mudança importante na percepção dos estudantes acerca dessas pessoas e de suas famílias e as palavras respeito e empatia apareceram frequentemente nos textos produzidos pelos estudantes. Aproximar os estudantes da realidade vivida pela pessoa com deficiência e de sua família parece ser uma alternativa para educar o estudante de Odontologia.
\end{abstract}

Descritores: Educação. Odontologia. Pessoas com Deficiência.

\section{INTRODUÇÃO}

Pessoa com deficiência (PD) é o termo utilizado para definir qualquer pessoa que tenha impedimentos de longo prazo de natureza física, mental, intelectual ou sensorial e que possam obstruir sua participação plena e efetiva na sociedade 
em igualdade de condições com as demais pessoas ${ }^{1}$. De acordo com o último censo demográfico realizado no Brasil, 23,9\% da população brasileira se encaixa nesta definição, o que equivale a aproximadamente 49 milhões de pessoas ${ }^{2}$, demandando dos órgãos governamentais ações visando implementar políticas de educação e saúde pública não apenas para as $\mathrm{PD}$, mas também para suas famílias.

Sob a ótica da Odontologia, algumas características dessas pessoas, tais como limitações físicas ou cognitivas, podem contribuir decisivamente para aumentar o risco do desenvolvimento de doenças bucais ${ }^{3}$, então é necessária a aproximação com seu núcleo familiar para promover e manter a sua saúde bucal. Em especial, para $\mathrm{PD}$, estabelecer um programa de colaboração mútua, conhecer a rotina das famílias é essencial para desenvolver habilidades nos estudantes. Uma vez que o currículo dos cursos é baseado fortemente em aulas pré-clínicas e exercícios laboratoriais, antes do atendimento do paciente propriamente dito ${ }^{4}$, não há um consenso entre as escolas de Odontologia sobre a melhor forma de ensinar aos estudantes essas habilidades que vão além do atendimento clínico ${ }^{5}$.

Na Pontifícia Universidade Católica do Paraná (PUCPR), localizada em Curitiba, desde a década de 1990 é ofertada aos estudantes uma disciplina denominada Clínica Integrada para Pacientes com Necessidades Especiais, que oferece assistência odontológica para PD. A despeito dos bons resultados obtidos, é claramente perceptível que os estudantes não se sentem confortáveis para o atendimento clínico no início de cada semestre letivo. Nesse ponto uma pergunta importante emerge: será que estudantes de Odontologia, recém-introduzidos na prática clínica, possuem habilidades para comunicar-se confortavelmente com pessoas com deficiência e com seus familiares? Dessa forma, o objetivo deste estudo foi avaliar a percepção dos estudantes de Odontologia a respeito das necessidades destas pessoas a partir da utilização de um cenário criado para tal finalidade.

\section{METODOLOGIA}

Trata-se de um estudo qualitativo transversal que foi desenvolvido no âmbito do projeto de formação de professores realizado pelo Centro de Ensino e Aprendizagem (CREARE) da PUCPR, submetido e aprovado pelo Comitê de Ética em Pesquisa da instituição (CAAE 65161317. 0.0000.0020). Após a aprovação, todos os participantes assinaram o Termo de Consentimento Livre e Esclarecido, consentindo sua participação no estudo. Participaram do estudo 60 acadêmicos de Odontologia regularmente matriculados na disciplina Clínica Integrada para Pacientes com Necessidades Especiais, que é ofertada a estudantes do último ano do curso de Odontologia da Pontifícia Universidade Católica do Paraná.

\section{Primeira atividade - formação dos grupos focais}

No primeiro encontro do semestre, os acadêmicos foram divididos aleatoriamente em 8 grupos focais e receberam um questionário semiestruturado com questões que exploraram as expectativas dos estudantes a respeito do atendimento de pessoas com deficiência e da rotina destas pessoas. As perguntas foram elaboradas pelos professores da disciplina de modo a estimular a discussão dentro de cada grupo a respeito das experiências e atitudes já vivenciadas pelos estudantes nas suas vidas pessoais e incluíram tópicos tais como: a. expectativas a respeito do atendimento de pessoas com deficiência; $b$. dificuldades que esperam encontrar; c. aspectos relevantes durante o atendimento clínico, entre 
outras perguntas. No final desta primeira etapa os estudantes foram orientados a formular duas perguntas que gostariam de fazer aos familiares ou a uma PD. A duração desta atividade, incluindo divisão em grupos, discussão, elaboração de respostas e das duas perguntas direcionadas aos familiares durou uma hora e trinta minutos.

\section{Segunda atividade - criação do cenário}

Logo após a atividade inicial, pessoas com deficiência e seus familiares, que haviam sido previamente contatados, foram convidados para entrar na sala de aula onde os estudantes estavam reunidos. Essas pessoas responderam às questões formuladas pelos estudantes e descreveram a sua rotina diária, seus anseios, dificuldades enfrentadas no dia-a-dia e suas experiências anteriores em atendimentos médicos e odontológicos. Os estudantes também tiveram a oportunidade de perguntar e interagir com as pessoas com deficiência, ouvir e compartilhar experiências, formular hipóteses sobre o atendimento clínico, e pensar sobre como melhorar o atendimento clínico à essas pessoas. Essa atividade durou duas horas.

\section{Terceira atividade - discussão entre os grupos}

Esta última atividade ocorreu no mesmo dia, logo após a atividade anterior. Nesta reunião, já sem a presença das famílias, os acadêmicos, novamente organizados em grupos, discutiram a respeito das experiências vividas durante o encontro com as pessoas com deficiência e seus familiares e responderam à duas perguntas finais: a. Houve mudança da sua percepção a respeito da pessoa com deficiência e seus familiares?; b. O que esse primeiro dia de aula acrescentou a você, sob o ponto de vista pessoal e profissional?

\section{Análise dos dados}

Os dados coletados nas três atividades foram sistemática e rigorosamente analisados e agrupados em categorias e temas de acordo com a percepção dos estudantes. Os dados obtidos foram organizados no pacote de software Atlas Ti para recuperação de dados qualitativos.

\section{RESULTADOS}

Os principais resultados obtidos foram agrupados em categorias de acordo com a percepção dos estudantes antes e depois da criação do cenário. A figura 1 revela a percepção dos estudantes antes da reunião. Observa-se que os estudantes se colocaram no centro da discussão argumentando que a pessoa com deficiência e seus familiares poderiam ser uma maneira importante para desenvolvimento das suas habilidades, tanto clínicas quanto de comunicação. A maioria dos estudantes também menciona que atender pessoas com deficiência não é sua prioridade profissional e apontam o medo, estresse, a dificuldade de comunicação e baixa recompensa financeira como motivos para tal, revelando falta de altruísmo.

Após a criação do cenário que envolveu pessoas com deficiência e familiares parece ter havido uma mudança importante na percepção dos estudantes acerca dessas pessoas e de suas famílias. A preocupação com a saúde geral do deficiente foi amplamente discutida e a palavra respeito apareceu inúmeras vezes nos textos produzidos pelos estudantes, respeito aos familiares e ao deficiente. Parece ter havido também uma diminuição da sensação de medo e do estresse que o atendimento do deficiente poderia ocasionar. Empatia e respeito foram as palavras que caracterizaram essa etapa da pesquisa (figura 2). 


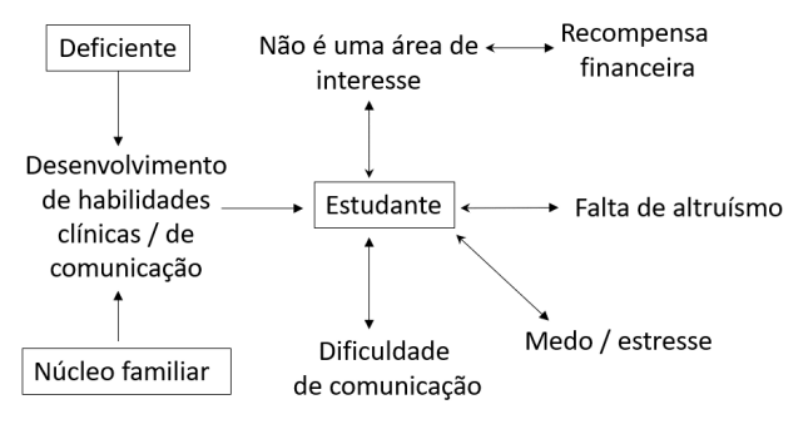

Figura 1. Estrutura conceitual delineada a partir das respostas dadas pelos estudantes antes do encontro com as pessoas com deficiência e seus familiares

\section{DISCUSSÃO}

Cursar Odontologia é um grande desafio para os estudantes uma vez que requer, além do conhecimento, desenvolvimento de habilidades que vão além daquelas denominadas clínicas ${ }^{6}$. Infelizmente, diversas situações de aprendizagem simplesmente não são contempladas no currículo, que normalmente se preocupa em desenvolver habilidades pré-clínicas laboratoriais baseadas em conhecimento teórico, sem o desenvolvimento de habilidades interpessoais ${ }^{7}$. Na prática, o estudante de Odontologia é colocado em situações de estresse constante e ainda não está devidamente preparado para isso. No que diz respeito ao atendimento de pessoas com deficiência, o nível de estresse pode aumentar bastante porque o estudante entende que a pessoa pode estar fragilizada.

Nesta pesquisa, estudantes de Odontologia, que nunca haviam atendido pacientes com deficiência, foram questionados sobre suas expectativas em relação aos atendimentos de pacientes com deficiência e colocados frente a frente com essas pessoas e seus familiares antes de executarem qualquer atendimento. Antes da conversa com os pais, dois pontos muito citados

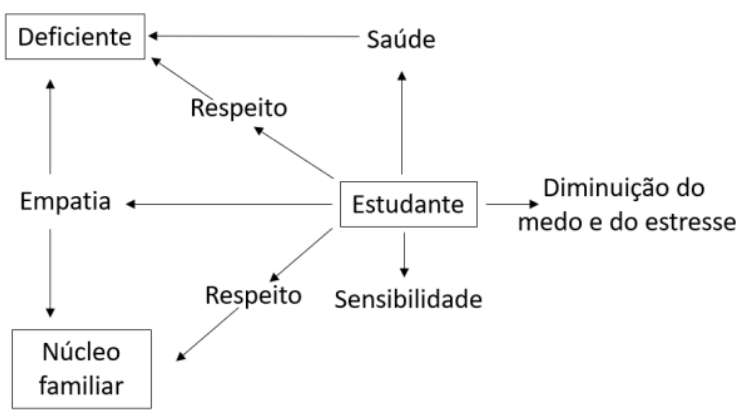

Figura 2. Estrutura conceitual delineada a partir das respostas dadas pelos estudantes após o encontro com as pessoas com deficiência e seus familiares

pelos estudantes foram a percepção de que haveria dificuldade de comunicação e, consequentemente dificuldade para realização de procedimentos clínicos nesta população, e o medo ou estresse no estudante desencadeado pelo atendimento de pessoas que, na opinião dos estudantes, são frágeis. No primeiro caso, vários estudantes mencionaram que teriam dificuldade em pedir aos pais detalhes sobre a deficiência de seus filhos evidenciando falta de habilidades de comunicação. No segundo caso, a maioria dos estudantes alegou não saber tratar clinicamente esses pacientes por causa de sua condição sistêmica enfraquecida. Curiosamente, outros estudos já mostraram que a percepção do estudante em odontologia em geral é que eles não estão adequadamente preparados para se comunicar com os pacientes ${ }^{8,9}$.

Uma pequena porcentagem de estudantes revelou não ter interesse no atendimento de deficientes porque não é economicamente viável, evidenciando uma certa falta de altruísmo ou desconhecimento das reais dificuldades enfrentadas por essas pessoas. Nesse ponto, a utilização de "pacientes educadores" pode ser uma ferramenta útil, porque traz inúmeros benefícios para a 
aprendizagem, entre eles retenção de conhecimento ${ }^{10}$. Nesta pesquisa, o cenário utilizado, com a presença de pessoas com deficiência e seus familiares assemelha-se muito com o papel desempenhado pelo paciente educador, proporcionado um "choque de realidade" nos estudantes, já que na reunião após a segunda atividade as palavras empatia e respeito surgiram inúmeras vezes.

De fato, a percepção dos estudantes parece ter mudado após o entendimento da condição de cada família/deficiente. Os estudantes parecem ter entendido que essas pessoas devem ser consideradas dentro de uma visão mais ampla, que envolve determinantes socioambientais e comportamentais ${ }^{6}$. Nesse contexto, surgiu inúmeras vezes a palavra empatia, que se exercitada, é uma virtude que pode modificar decisivamente a relação entre o cirurgiãodentista e seus pacientes ${ }^{11}$. Por outro lado, é uma obrigação das escolas de Odontologia desenvolver estratégias para entregar profissionais com habilidade suficiente para entender necessidades específicas de cada população ${ }^{5}$.

Outro dado interessante observado foi que, depois de conversar com os familiares, a percepção da fragilidade da pessoa com deficiência parece ter mudado. Os relatos do grupo mencionam que a confiança transmitida pelos pais ao falar sobre as necessidades de seus filhos reduziu a impressão de que as pessoas com deficiência são frágeis e elevou a confiança dos estudantes. Também as palavras estresse e ansiedade, muito mencionadas pelos estudantes no início das atividades, foram praticamente esquecidas após a reunião com os pais, indicando que conhecer a rotina da família e da pessoa com deficiência parece ser uma alternativa para amenizar o estresse desses estudantes.

É notório que existem desigualdades em saúde bucal para pessoas com deficiência, sejam elas crianças, jovens ou adultos, homens ou mulheres, mas também deve ser considerado que o estudante de Odontologia também sofre consequências psicológicas e emocionais pelo fato de não estar tão bem preparado para lidar com pessoas com deficiência, o que pode ser desastroso, do ponto de vista pessoal e profissional. Da mesma forma, aproximar o estudante da realidade vivida pela pessoa com deficiência e de sua família pode ser uma alternativa viável e fácil de ser introduzida. Talvez seja uma alternativa para modificar os currículos dos cursos de Odontologia que atendam pessoas com deficiência.

\section{CONCLUSÃO}

Este estudo mostrou que aproximar os estudantes de Odontologia das pessoas com deficiência e de seus familiares, antes do atendimento clínico propriamente dito, mudou a percepção dos mesmos sobre essas pessoas e parece ser uma alternativa para educar e formar os estudantes.

\section{AGRADECIMENTOS}

Esta pesquisa foi apoiada pela FINEP e desenvolvida como parte do projeto de formação de professores realizado pelo Centro de Ensino e Aprendizagem (CREARE) da PUCPR.

\section{ABSTRACT \\ Use of scenarios with people with disabilities and their families in dental education}

The aim of this study was to use a scenario involving people with disabilities, their families, and dental students to assess students' perceptions of the needs of people with disabilities. This is a qualitative crosssectional study that included 60 dental students enrolled in the discipline of Integrated Clinical Care of Patients with Special Needs. Focus groups were formed and each group received a semi-structured 
questionnaire with questions about aspects of the care provided to people with disabilities and about their routine. A scenario was then used in which students interacted with people with disabilities and their families. In the last phase of the study, the groups formulated hypotheses about the care of these patients. The data collected in three time periods were grouped into categories and themes and analyzed using the Atlas Ti software. The results obtained were categorized according to the students' perception before and after the creation of the scenario. After the qualitative analysis, the data revealed that, before the creation of the scenario, the students placed themselves at the center of the discussion, arguing that attending to people with disabilities would be an opportunity to develop their skills without worrying about people with disabilities themselves. After the creation of the scenario, there seems to have been an important change in the students' perception of these people and of their families and the words 'respect' and 'empathy' appeared frequently in the texts produced by the students. Bringing students closer to the reality experienced by people with disabilities and their families seems to be an alternative to educate dental students.

Descriptors: Education. Dentistry. Disabled Persons.

\section{REFERÊNCIAS}

1. Ministério da Saúde. Atenção à saúde da pessoa com deficiência no sistema único de saúde SUS. 2009;(1):33.

2. Censo Demográfico 2010. Características da população e dos domicílios: resultados do universo. Rio de Janeiro: IBGE, 2011. 270 p. [Acesso em 30 de julho de 2019]. Disponível em: https://biblioteca.ibge.gov.br/visualizacao/p eriodicos/94/cd_2010_religiao_deficiencia.pdf.

3. Shin CJ, Saeed S. Toothbrushing barriers for people with developmental disabilities: a pilot study. Spec Care Dent. 2013;33(6):269-74.

4. Olowo-Ofayoku A, John Moxham B.
Comparisons between the attitudes of medical and dental students toward the clinical importance of gross anatomy and physiology. Clin Anat. 2014;27(7):976-87.

5. Donate-Bartfield E, Lobb WK, Roucka TM. Teaching culturally sensitive care to dental students: a multidisciplinary approach. J Dent Educ. 2014;78(3):454-64.

6. Tubert-Jeannin S, Jourdan D. Renovating dental education: a public health issue. Eur $\mathbf{J}$ Dent Educ. 2018;22(3):e644-e47.

7. Lown Ba, Sasson JP, Hinrichs P. Patients as partners in radiology education. an innovative approach to teaching and assessing patientcentered communication. Acad Radiol. 2008;15(4):425-32.

8. Postma TC, White JG. Developing students' clinical reasoning skills: correlates of perceived relevance of two teaching and learning approaches. Eur J Dent Educ. 2017;21(1):52-57.

9. Botelho M, Gao X, Bhuyan SY. An analysis of clinical transition stresses experienced by dental students: a qualitative methods approach. Eur J Dent Educ. 2018;22 (3):e564-e72.

10. Edwards PC, Graham J, Oling R, Frantz KE. The patient educator presentation in dental education: reinforcing the importance of learning about rare conditions. J Dent Educ. 2016;80(5):533-41.

11. Bark MM, Posanski M, Oliveira KV, Brancher JA, Kriger L, Gabardo MCL. Alterity and empathy : essential virtues for the training of the dentist. Rev ABENO. 2018;18:104-13.

\section{Correspondência para:}

João Armando Brancher

e-mail: brancher.a@gmail.com

Rua Imaculada Conceição, 1155 - Prado Velho 80215-901 Curitiba/PR 